\title{
Blocking Probability Analysis of Wireless Sensors That Employ Opportunistic Spectrum Access
}

\author{
L. Anusha ${ }^{\#}$, B.Seetha Ramanjaneyulü, K. Annapurna ${ }^{\#}$ \\ \# Department of ECE, VFSTR University, Vadlamudi, Guntur, 522213, India \\ E-mail:anusha.lingineni@gmail.com,ramanbs@gmail.com,arya.anu85@gmail.com
}

\begin{abstract}
Opportunistic accessing of vacant channels of licensed spectrum by non-licensed wireless devices offers a convenient solution to the spectrum scarcity problems experienced by many wireless communication systems. Due to the fixed assignment policy of spectrum, some of the licensed frequencies are not in full utilization by their licensed users. On the other hand, there exists a severe scarcity of bandwidth for new wireless services. In this context, opportunistic access is found to be a boon, to overcome the spectrum scarcity problems. In this paper, opportunistic accessing of vacant channels by the wireless sensors is analysed. Finding the number of devices that can be supported for the available vacant bandwidth is the main focus of the work. Assessing the blocking probabilities experienced by the devices is found to offer an approximation of the number of devices that can be accommodated in a given scenario, for permissible blocking rates. This kind of analysis can help in proper planning of such wireless sensor networks, to deploy them in a way that they can make use of the white space bandwidths efficiently.
\end{abstract}

Keywords—: WSN; Spectrum Access; Spectrum White Spaces; Interference Mitigation; Opportunistic Access.

\section{INTRODUCTION}

Since there is a rapid growth in the wireless communication technologies and services, wireless devices are used for many applications. All these applications require bandwidth allocations for their operation, which increases the demand of wireless spectrum. But the wireless spectrum resources are limited. Efficient spectrum management has been proposed by regulators like Federal Communications Commission (FCC) for both licensed users and unlicensed uses [1]. Recent spectrum measurements reveal that the spectrum that is allotted to licensed users is largely unutilized and many channels of the spectrum allocated to some users, remained vacant. Such vacant channels can be accessed by using cognitive radio dynamic spectrum access methods [2]. FCC permits sharing of licensed user's spectrum by the unlicensed users without causing interference. Opportunistic accessing of vacant channels increases the efficiency of spectrum utilization and reduces the problem of spectrum scarcity.

Cognitive radio techniques allow many devices to use the licensed spectrum without having exclusive license to access. Wireless sensors are power constrained devices and they operate in ISM bands like the $900 \mathrm{MHz}$ and $2.4 \mathrm{GHz}$ bands. As they are used for many applications, the available spectrum in ISM bands is not enough for their transmission needs, which results in data loss [3]. In this context, They are adopting the cognitive radio methods with opportunistic accessing of vacant channels to overcome the spectrum scarcity. Wireless channels are subjected to path fading, interference, and shadow fading. Hence, the state of wireless channels varies with time slots, frequencies and geographical locations. By taking all the above considerations into account, an analysis is carried out in this paper, to find the number of sensors that the available vacant channel can accommodate, within the acceptable blocking rates. Section2 of the paper discusses about the dynamic spectrum access mechanism and its adoption to wireless sensor networks. Section-3 discusses about the proposed model of network and its simulation results. Section-4 concludes the paper.

\section{DyNAMIC SPECTRUM ACCESS By WIRELESS SENSOR NETWORKS}

Dynamic spectrum access was one of the solutions for the growing bandwidth needs of the wireless devices. This technique was introduced by Joseph Mitola [4]. It proposes to use the licensed spectrum by unlicensed users also, without causing interference. Licensed users are known as primary users and unlicensed opportunistic users are known as secondary users, in this system. It can be implemented with overlay or underlay mechanisms. In overlay cognitive radio system, the secondary users will use the channels only when the primary users do not use them In the case of underlay cognitive radio system, the secondary 
users coexist along with primary users, in such a way that their presence is not felt by the primary users [6]. In this later case, the secondary users operate with lesser power levels and suitable interference mitigation methods such that their transmissions are not affected by primary users' power levels.

In its early proposals, cognitive radio techniques were proposed mainly for providing data services to wireless users. Of late, they were extended to be used with vehicular networks, and sensor networks which depend on unlicensed ISM bands mainly. The reason for this extension was because of the crowding in ISM bands due to the increased number of devices that use these unlicensed bands. So, dynamic spectrum access methods were proposed to these devices also [7].

To implement dynamic spectrum access, the device should at first know which channel is vacant. This can be known by sensing the spectrum or getting that information from another source like database or a nearby device. Sensing the spectrum is a complex and power consuming task, which cannot be handled by tiny devices like sensors. So, when dynamic spectrum accessing is proposed to be used with sensors, the spectrum sensing may not be carried out by each sensor. Instead, a few devices that are capable of performing the spectrum sensing do that job and share that information to all the sensors. Sometimes, information stored in databases also will be helpful to offer a detail of which channels could be vacant in the given geographical location. Through this information, the local sensing will be limited to those channels only, which can reduce the sensing burden.

One important thing that needs to be fulfilled here is that the sensor device should be capable of transmitting in different frequencies that become vacant from time-to-time. In general, the wireless sensors that use ISM bands are capable of transmitting the $900 \mathrm{MHz}$ and $2.4 \mathrm{GHz}$ ISM bands, which are of about $30 \mathrm{MHZ}$ and $80 \mathrm{MHZ}$ wide respectively. Now, when the device has to use the available vacant channel of the spectrum, mostly the UHF frequencies of TV channels, the device should be equipped with transceivers that are capable of operating in these frequencies. However, these days, with hardware costs coming down heavily, this requirement is not a limitation.

\section{PROPOSED SYSTEM AND SIMULATION RESULTS}

To carry out the proposed analysis, a network system with varying number of vacant channels and varying number of sensor devices is considered. Each channel is considered to be having $5 \mathrm{MHz}$ bandwidth. The device that wants to use this channel can request for $1 \mathrm{MHz}$ or multiples of it upto 5 $\mathrm{MHz}$ from the system. Three different scenarios of wireless sensors with 30, 60 and 90 sensors is considered for the WSN. The sensor may not need continuous channel access. The number of times it requires the access and the duration it wants to use the channel in each of its opportunistic access also play an important role.

Simulation is carried out here for different number of access requests and different channel durations the sensor device needs.

\section{A. Scenario-1}

The first scenario is for 20 requests by each device for an average usage duration of 200 units of time, within the simulation duration of 1440 units of time. Availability of free channels is considered as two, totalling to $10 \mathrm{MHz}$ bandwidth. As can be expected, many sensors will not get the channel access opportunity, for this scenario. These results are shown in Fig-1, 2 and 3. The results are presented in terms of the number of requests from sensors, average blocking probability and number of sensors that got service.

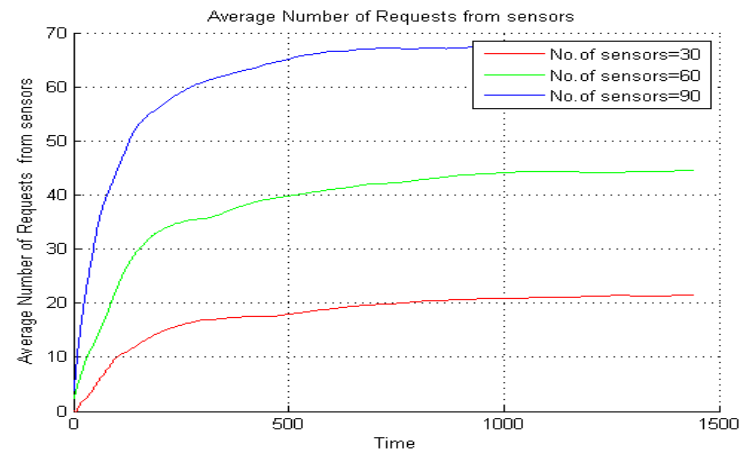

Fig.1 Average Number of Requests from Sensors in Scenario -1

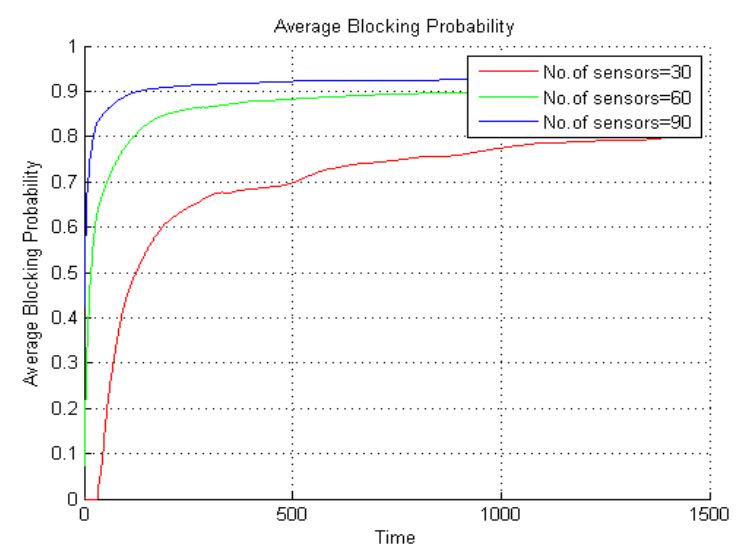

Fig. 2 Average Blocking Probability in scenario -1

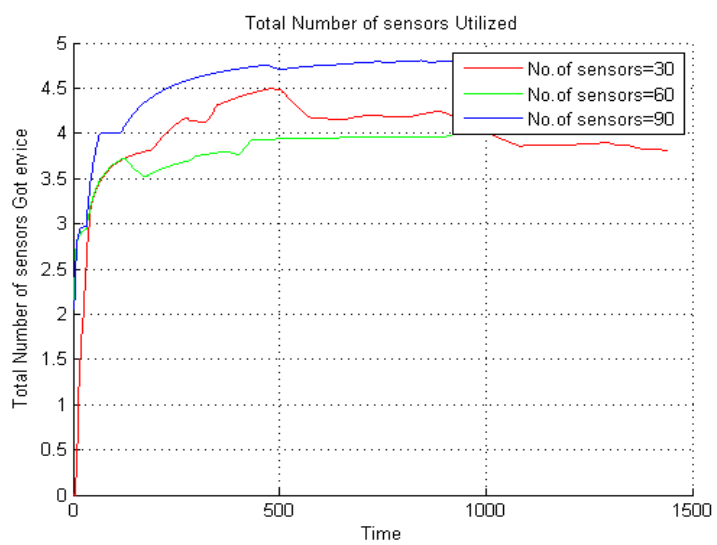

Fig.3 Total number of sensors got service in scenario-1

From the above result it is observed that blocking probabilities are in the range of $0.8,0.9$ and 0.95 for the cases of 30, 60 and 90 sensors. These values of blocking probabilities are considered to be on the higher side. So, it can be concluded that the available bandwidth cannot support this scenario. 


\section{B. Scenario-2}

The second scenario is also for 20 requests by each device for an average usage duration of 200 units of time, within the simulation duration of 1440 units of time. Number of available channels considered here is 5, totalling to 25 $\mathrm{MHz}$ bandwidth. Simulation results are presented in Fig-4, 5 and 6.

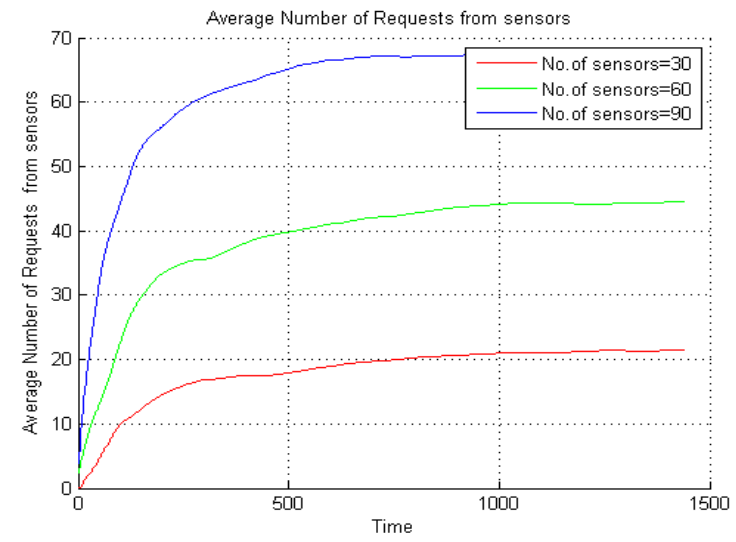

Fig.4 Average Number of Requests from Sensors in Scenario -2

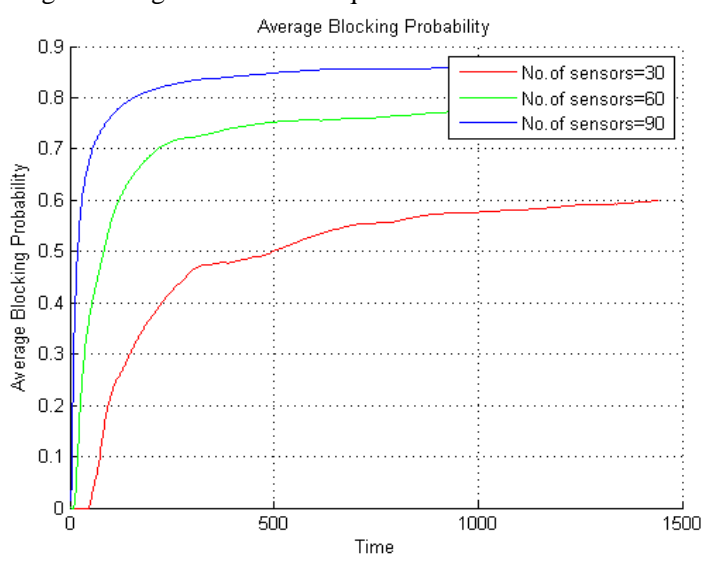

Fig.5 Average Blocking Probability in scenario-2

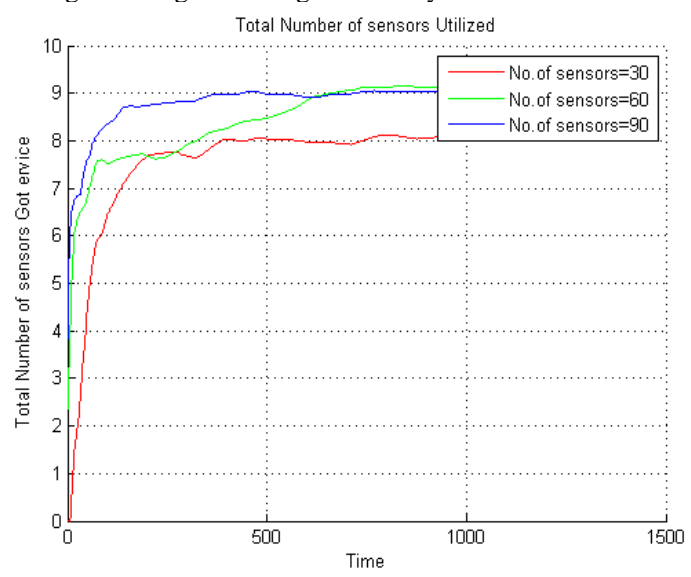

Fig.6 Total number of sensors got service in scenario-2

From the above results, it is observed that blocking probabilities are in the range of $0.6,0.8$ and 0.95 for the cases of 30, 60 and 90 sensors. These values of blocking probabilities are still on the higher side and hence it can be concluded that the available bandwidth cannot support this scenario also.

\section{Scenario-3}

The third scenario is for 30,60 , and 90 sensors again, but the number of available vacant channels is considered as 8. The number of requests from each device is taken as 10 and average usage duration is taken as 100 units of time. That means, data transmission requirements are one-fourth of the previous two scenarios. Results are presented in Fig-7, 8 and 9 .

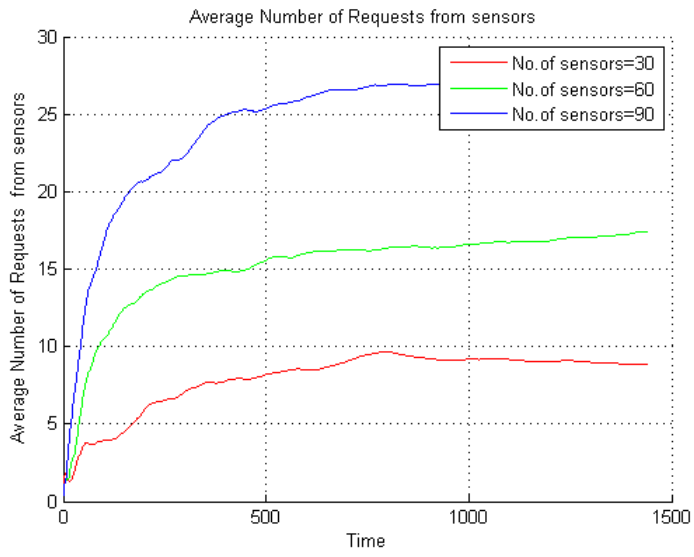

Fig.7 Average Number of Requests from Sensors in Scenario -3

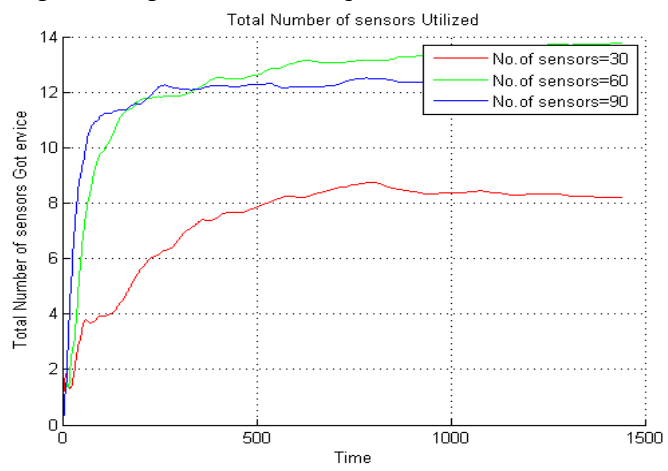

Fig.8 Average Blocking Probability in scenario-3

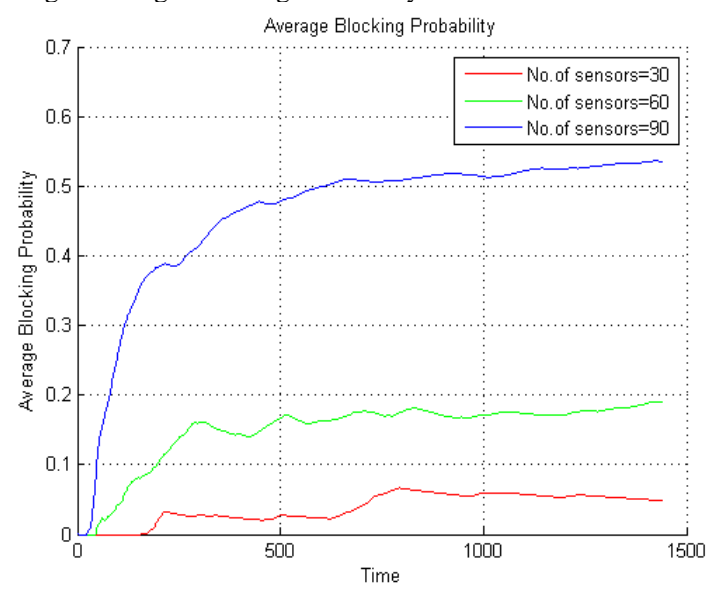

Fig.9 Total number of sensors got service in scenario-3

From the above results, it is observed that blocking probabilities are in the range of $0.05,0.2$ and 0.5 for the cases of 30,60 and 90 sensors. These values of blocking probabilities indicate that 30 sensors can easily be accommodated for the available bandwidth here. 60 sensors also can be accommodated, if real-time constraints are not there for sensor data transmissions. 


\section{Scenario-4}

The fourth scenario is for 30, 60 and 90 sensors again, but the numbers of available vacant channels are 10, and number of requests from each device is 10 and average usage duration is 100 units of time. As expected more number of devices can get service in this scenario. Results are presented in Fig-10,11,12.

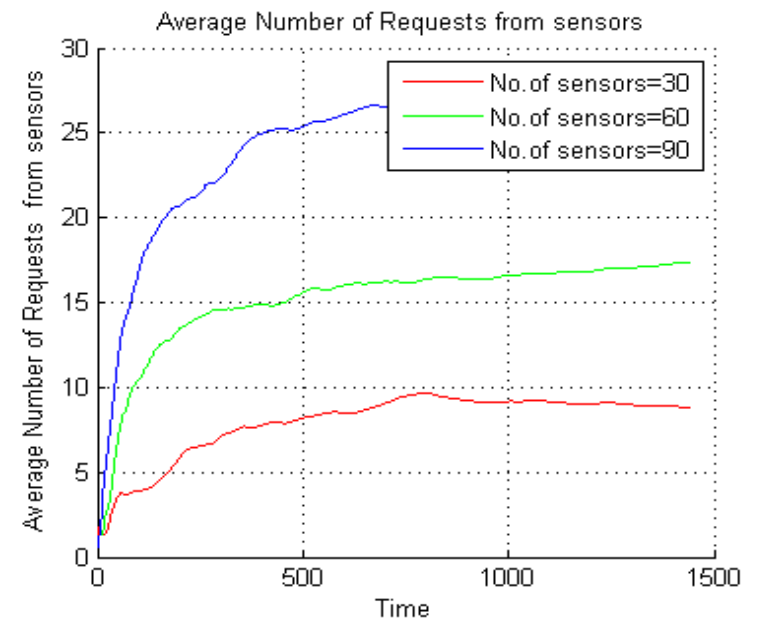

Fig.10 Average Number of Requests from Sensors in Scenario -4

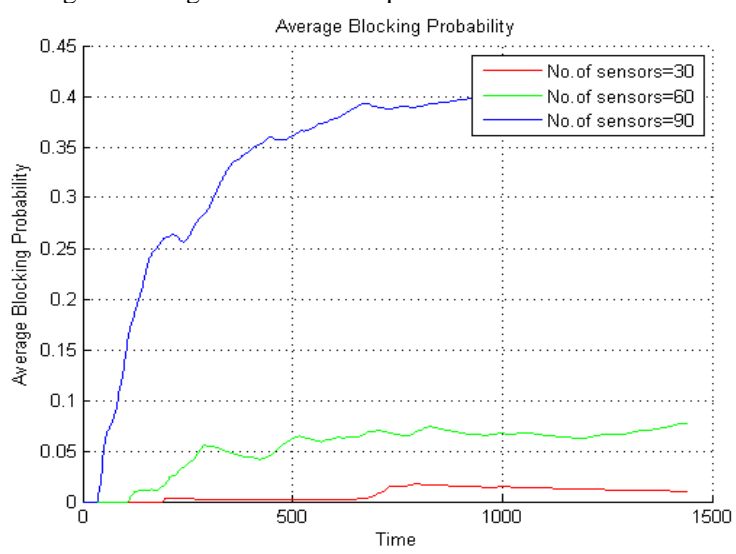

Fig.11 Average Blocking Probability in scenario-4

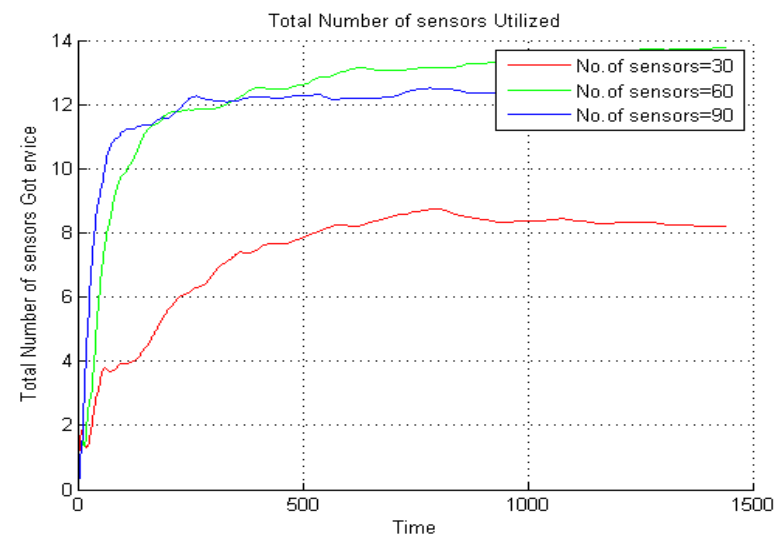

Fig.12 Total number of sensors got service in scenario- 4

From the results of scenario-4, it is observed that blocking probabilities are in the range of $0.02,0.05$ and 0.4 for the cases of 30, 60 and 90 sensors. These values of blocking probabilities indicate that for the cases of 30 sensors almost all the sensors which are requesting for channels can get the service. 60 sensors also can be easily accommodated with the available bandwidth. For the case of 90, it can support if real time constraints are not there for the devices. If realtime constraints are there to some of the devices, prioritized access can be offered to such devices.

\section{CONCLUSION}

As the unlicensed ISM bands have become crowded, sensor networks also started exploring the use of opportunistic channel access methods, to use licensed vacant channels for the transmission needs of the sensor nodes. In this work, analysis is carried out to find out the number of sensors that can be accommodated by the available vacant channel bandwidths. Various scenarios of the system, with varying number of nodes, varying channel bandwidth availability, and varying data transmission needs of the devices are simulated here. Through this simulation, blocking probabilities of various situations are found. This helps in choosing the required planning of WSN with supporting number of devices that can be formed into a network. This makes it possible to divide the network into required number of sub networks where the frequencies can be reused after short distances in the same region. This will be possible with appropriate power control mechanisms implemented with the devices.

\section{REFERENCES}

[1] FCC-NPRM, (2009) In the matter of amendment of the commission's rules to provide spectrum for the operation of medical body area networks, FCC 09-57.

[2] Q. Zhao and B. M. Sadler, "A Survey of Dynamic Spectrum Access," in IEEE Signal Processing Magazine, vol. 24, no. 3, pp. 79-89, May 2007.

[3] A. Ahmad, S. Ahmad, M. H. Rehmani and N. U. Hassan, "A Survey on Radio Resource Allocation in Cognitive Radio Sensor Networks," in IEEE Communications Surveys \& Tutorials, vol. 17, no. 2, pp. 888- 917, Secondquarter 2015.

[4] J. Mitola and G. Q. Maguire, "Cognitive radio: making software radios more personal," in IEEE Personal Communications, vol. 6, no. 4, pp. 13-18, Aug 1999.

[5] Mitola J, "Cognitive Radio Architecture Evolution", Proceedings of IEEE, Vol.97, No.4, pp. 626-641, 2009.

[6] Ian F. Akyildiz, Won-Yeol Lee, Mehmet C. Vuran, Shantidev Mohanty ,"Next generation/dynamic spectrum access/cognitive radio wireless networks: A survey", Elsevier's Journal of Computer Networks, Vol.50,2127-2159, September 2006

[7] Yiping Xing, Chandramouli R, Mangold S, "Dynamic Spectrum Access in Open Spectrum Wireless Networks", IEEE Journal on Selected Areas In Communications, VOL. 24, NO. 3, , pp. $626-$ 637, 2006

[8] Ian F. Akyildiz, Won-Yeol Lee, Mehmet C. Vuran, and Shantidev Mohanty, "A Survey on Spectrum Management in Cognitive Radio Networks", IEEE Communications, 2008, pp. 40-48

[9] O. B. Akan, O. B. Karli and O. Ergul, "Cognitive radio sensor networks," in IEEE Network, vol. 23, no. 4, pp. 34-40, July-August 2009.

[10] M. Askari, Y. S. Kavian, H. Kaabi and H. F. Rashvand, "A channel assignment algorithm for Cognitive Radio wireless sensor networks," IET Conference on Wireless Sensor Systems (WSS 2012), London, 2012, pp. 1-4.

[11] S. S. Byun, I. Balasingham and X. Liang, "Dynamic Spectrum Allocation in Wireless Cognitive Sensor Networks: Improving Fairness and Energy Efficiency," 2008 IEEE 68th Vehicular Technology Conference, Calgary, BC, 2008, pp. 1-5

[12] M. Cardei and A. Mihnea, "Channel assignment in cognitive wireless sensor networks," 2014 International Conference on Computing, 
Networking and Communications (ICNC), Honolulu, HI, 2014, pp. 588-593

[13] D. Cavalcanti, S. Das, J. Wang and K. Challapali, "Cognitive Radio Based Wireless Sensor Networks," 2008 Proceedings of 17th International Conference on Computer Communications and Networks, St. Thomas, US Virgin Islands, 2008, pp. 1-6

[14] S. Gao, L. Qian and D. R. Vaman, "Distributed Energy Efficient Spectrum Access in Wireless Cognitive Radio Sensor Networks," 2008 IEEE Wireless Communications and Networking Conference, Las Vegas, NV, 2008, pp. 1442-1447.

[15] Jamal, C. K. Tham and W. C. Wong, "Event detection and channel allocation in cognitive radio sensor networks," 2012 IEEE International Conference on Communication Systems (ICCS), Singapore, 2012, pp. 157-161.

[16] S. Maleki, A. Pandharipande and G. Leus, "Energy-Efficient Distributed Spectrum Sensing for Cognitive Sensor Networks," in IEEE Sensors Journal, vol. 11, no. 3, pp. 565-573, March 2011.

[17] K. Shenai and S. Mukhopadhyay, "Cognitive sensor networks," 2008 26th International Conference on Microelectronics, Nis, 2008, pp. 315-320.

[18] P. Spachos, P. Chatzimisios and D. Hatzinakos, "Cognitive networking with opportunistic routing in Wireless Sensor
Networks," 2013 IEEE International Conference on Communications (ICC), Budapest, 2013, pp. 2433-2437.

[19] G. Vijay, E. Bdira and M. Ibnkahla, "Cognitive approaches in Wireless Sensor Networks: A survey," 2010 25th Biennial Symposium on Communications, Kingston, ON, 2010, pp. 177-180

[20] J. Mitola III and H. Man, "Semantics in Cognitive Radio," 2009 IEEE International Conference on Semantic Computing, Berkeley, CA, 2009, pp. 261-266.

[21] M. H. Rehmani, S. Lohier and A. Rachedi, "Channel bonding in cognitive radio wireless sensor networks," 2012 International Conference on Selected Topics in Mobile and Wireless Networking, Avignon, 2012, pp. 72-76.

[22] P. Spachos, P. Chatzimisios and D. Hatzinakos, "Cognitive networking with opportunistic routing in Wireless Sensor Networks," 2013 IEEE International Conference on Communications (ICC), Budapest, 2013, pp. 2433-2437.

[23] K. L. A. Yau, P. Komisarczuk and P. D. Teal, "Cognitive Radiobased Wireless Sensor Networks: Conceptual design and open issues," 2009 IEEE 34th Conference on Local Computer Networks, Zurich, 2009, pp. 955-962

[24] Y. SUN, "Distributed fast channel allocation in cognitive wireless sensor networks," in IET Signal Processing, vol. 10, no. 5, pp. 471477, 72016. 